\title{
Preparation of silk fibroin-poly(ethylene glycol) conjugate films through click chemistry
}

\author{
Sandra Sampaio," Teresa M. R. Miranda, Jorge G. Santos \\ and Graça M. B. Soares
}

\begin{abstract}
Azide silk fibroin (azido SF) and alkyne terminal poly(ethylene glycol) (PEG) 2000 (acetylene-terminal PEG 2000) were synthesized. Azido SF was reacted with acetyleneterminal PEG 2000 to produce films via a copper-mediated 1,3-cycloaddition ('click' chemistry) generating a triazole linkage as the networking forming reaction. Through click chemistry, novel silk-based films with various weight ratios were prepared and investigated. Fourier transform infrared, $\mathrm{X}$-ray diffraction and differential scanning calorimetry analyses showed that the ordered association of the PEG molecules is strongly constrained by the presence of the SF molecules and crosslinking and that the presence of acetylene-terminal PEG 2000 in the films induced crystallization to a $\beta$-sheet of SF chains.Water content and contact angle measurements indicated that the hydrophilicity of the films increased compared with SF. SF-PEG films exhibited smooth and rough structures, depending on degree of crosslinking and on the weight ratio of SF and PEG, as shown by scanning electron microscopy.
\end{abstract}

Keywords: silk fibroin; films; click chemistry; poly(ethylene glycol) 